\title{
NECESSARY THERMODYNAMICS FACTORS TO OBTAIN SIMPLE SOLID SOLUTIONS IN HIGH-ENTROPY ALLOYS FROM THE Al-Ti-Co-Ni-Fe SYSTEM
}

\begin{abstract}
In this paper findings regarding the design and synthesis of High-Entropy Alloys based on mixing enthalpy, mixing entropy, $\delta$ parameter, $\Omega$ parameter and valence electron concentration are presented. Four alloys were synthesised with different predicted crystalline structures. Results of the microstructure and crystal structure studies are presented. It was shown that predicted structures as well as complex intermetallic phases exist in the material. The validity of valence electron concentration as well as additional parameters such as mixing enthalpy, mixing entropy and others necessary to obtain only the solid solution in High-Entropy Alloys were examined.
\end{abstract}

Keywords: High-Entropy Alloys, Entropy, Enthalpy, VEC, solid solution

\section{Introduction}

Recently, one of the most popular and evolving group of materials in materials science are high-entropy alloys (HEA). According to the definition, these alloys contain at least five principal metallic elements (5-35 at. pct. each) [1-4]. This group is characterized by high hardness, wear resistance, high temperature resistance, and oxidation resistance [5-12]. High entropy alloys are expected to form solid solutions with simple crystallographic structures such as FCC and/or BCC [13-15]. According to the Guo et al. [14], the crystallographic structure and the stability of the solid solutions may be successfully predicted by the use of valance electron concentration (VEC). The total number of d-electrons in the valence band of each constituent element $\left(V E C_{i}\right)$ and its molar ratio $\left(c_{i}\right)$ are used for $V E C$ calculation for the multi-component alloys (Eq. 1).

$$
V E C=\sum c_{i} V E C_{i}
$$

It was assumed by Guo et al. [14] that VEC lower than or equal to 6.87 results the $\mathrm{BCC}$ crystal structure, whereas values greater than or equal to 8 results the FCC crystal structure. When the $V E C$ parameter is between 6.87 and 8 , dual phase structure exists.

The objective of this work is to compare the calculated $V E C$ parameters of selected alloys with the theoretical assumptions and experimental results. Aluminum, titanium, cobalt, nickel, and iron were chosen as an alloying elements in the experiments.
The chemical composition of each alloy was designed to obtain different crystallographic structures. The $V E C_{i}$ value used for calculations and crystal structure of each element is presented in the Table 1. Aluminum and titanium were chosen to stabilize the BCC phase $\left(V E C_{i}<6.87\right)$ while cobalt, nickel and iron were used to stabilize the FCC phase $\left(V E C_{i} \geq 8\right)$.

TABLE 1

The value of $V E C$ and crystallographic structure for chosen elements

\begin{tabular}{|c|c|c|c|c|c|}
\hline \hline Element & Al & Ti & Co & Ni & Fe \\
\hline$V E C_{i}$ & 3 & 4 & 9 & 10 & 8 \\
\hline $\begin{array}{c}\text { Crystallographic } \\
\text { structure of element }\end{array}$ & FCC & HCP & HCP & FCC & BCC \\
\hline
\end{tabular}

\section{Experimental}

The investigated alloys from the Al-Ti-Co-Ni-Fe system were synthesized by an arc melting of pure elements in the Ti-gettered argon atmosphere on a water-cooled copper mould (AM Edmund Bühler). In order to improve the homogeneity of the alloy, the obtained samples were re-melted at least four times. The chemical composition of each alloy was designed to obtain different crystal structures - BCC, BCC+FCC, and FCC (Table 2), predicted on the basis of the calculated VEC value. For further investigations, middle sections of ingots were chosen for the preparation of specimens. Obtained samples were ex-

\footnotetext{
AGH UNIVERSITY OF SCIENCE AND TECHNOLOGY, FACULTY OF METALS ENGINEERING AND INDUSTRIAL COMPUTER SCIENCE, DEPARTMENT OF PHYSICAL AND POWDER METALLURGY, AL. MICKIEWICZA 30, 30-059 KRAKOW, POLAND

** AGH UNIVERSITY OF SCIENCE AND TECHNOLOGY, ACADEMIC CENTRE FOR MATERIALS AND NANOTECHNOLOGY, AL. MICKIEWICZA 30, 30-059 KRAKOW, POLAND

\# Corresponding author: kgorecki@agh.edu.pl
} 
pected to represent the most homogenous chemical composition. Samples were polished and etched with $\mathrm{HCl}+\mathrm{HNO}_{3}+\mathrm{CH}_{3} \mathrm{OH}$ (100:3:100).

The microstructures of each alloy were investigated by the use of scanning electron microscopy (SEM, FEI Versa 3D). The crystalline structure of investigated samples was identified by XRD measurements (XRD Panalytical Empyrean using Co K $\alpha 1$ radiation $(\lambda=1.789 \AA)$.

TABLE 2

Chemical composition atomic pct., calculated $V E C, \Delta H_{m i x}, \Delta S_{m i x}$ and crystal lattice of synthesized alloys

\begin{tabular}{|c|c|c|c|c|c|c|c|c|c|}
\hline \hline & $\mathbf{A l}$ & $\mathbf{T i}$ & $\mathbf{C o}$ & $\mathbf{N i}$ & $\mathbf{F e}$ & $\begin{array}{c}\Delta \boldsymbol{H}_{\text {mix }}, \\
{\left[\mathbf{k J} / \mathbf{m o l}^{-1}\right]}\end{array}$ & $\boldsymbol{V E C}$ & $\begin{array}{c}\text { Predicted } \\
\text { crystal lattice }\end{array}$ & $\begin{array}{c}\Delta \boldsymbol{S}_{\text {mix }}, \\
{[\mathbf{J} / \mathbf{m o l} \cdot \mathbf{K}]}\end{array}$ \\
\hline 1. & 30 & 30 & 10 & 10 & 20 & -30.2 & 5.60 & BCC & 12.5 \\
\hline 2. & 15 & 20 & 20 & 25 & 20 & -25.3 & 7.15 & FCC+BCC & 13.3 \\
\hline 3. & 10 & 10 & 25 & 35 & 20 & -16.9 & 8.05 & FCC & 12.4 \\
\hline 4. & 5 & 5 & 35 & 35 & 20 & -9.5 & 8.6 & FCC & 11.3 \\
\hline
\end{tabular}

\section{Results}

As was already mentioned in Part 2 that each alloy was designed to obtain a specific crystal lattice structure. According to the calculated values of the $V E C$ parameter, alloys 1, 3 and 4 should form a solid solution of only one type of structure, BCC or FCC. On the other hand, alloy 2 is expected to have both FCC and BCC structures. The negative value of $\Delta H_{\text {mix }}$ means that components were thoroughly mixed together in the formed solid solutions. If $\Delta H_{m i x}$ would be positive it will be impossible to create a solution (no mixing in solid state). Both parameters, $V E C$ and $\Delta S_{m i x}$ (except of alloy 4), fulfil the formal definition of HEA, thus the investigated alloys were examined as HighEntropy Alloys.

The microstructure and the phase composition of all investigated alloys are presented in the Fig. 1. The microstructure of alloy 1 (Fig. 1A) is characteristic for microstructures of as-cast samples. The dendrites (light) and interdendritic areas (dark) form elongated areas. In comparison to other synthesised samples, alloy 1 also has imperfections (such as cracks) visible in Fig. 1A. The XRD measurement performed on alloy 1 (Fig. 1A') reveals three different $\mathrm{BCC}$ phases and $\mathrm{AlFeTi}$-like Laves phase.

The microstructure of alloy 2 (Fig. 1B) is characterized by the occurrence of dendrites (dark) surrounded by the interdendritic areas (light) and eutectic. This kind of formation of dendrites and interdendritic areas also named "flower-like" is not usual in conventional (two or three elements) alloys. In the ID (interdendritic region), irregular eutectic was noticed. The eutectic forms the discontinuous formations in ID. The analysis of the XRD pattern (Fig. 1B') enables the identification of three types of phases: 2 BCC-type, 1 FCC-type, and the BCT-type.

The microstructure of alloy 3 (Fig. 1C) consists of large dendrites (light), and the interdendritic space (dark). Two FCC phases were identified in this alloy.
In addition, the microstructure of alloy 4 consists of dendrites, yet they are much smaller than in other alloys (Fig. 1D), and small interdendritic space. There were also indexed two FCC phases (Fig. 1D').

\section{Discussion}

Results presented in this paper show that neither of investigated alloys have the crystal structure predicted on the basis of $V E C$. Taking $V E C$ into account, alloys 1, 3 and 4 should form single-phase structures, and alloy 2 should form dualphase structure. Apart from BCC and FCC phases present in the material, additional phases such as Laves-type, BCT have been identified. This kind of alloys are susceptible to cracking, due to the occurrence of Laves-type phase in alloy 1 and eutectic in alloy 2 . In the case of alloy 4 the existence of FCC2 phase is the result of element substitution in the material. In the discussed system constituent elements tend to segregate, which could result in forming similar phases (solid solutions) but formed from different elements - it is possible because of probably substitutions of elements. A good example of this is alloy 4, where titanium trend to segregate to dendrites boundary and interdendritic spaces (Fig. 2).

In order to correctly estimate the HEA phase composition not only should $V E C$ be taken into consideration, but also another parameters such as $\Delta S_{m i x}$ (eq. 2), $\Delta H_{m i x}$ (eq. 3):

$$
\Delta S_{\text {mix }}=-R \sum c_{i} \ln c_{i}
$$

where: $R$-gas constant $(8.31 \mathrm{~J} / \mathrm{mol} \cdot \mathrm{K}), c_{i}-$ molar ratio of each element.

$$
\Delta H_{\text {mix }}=\sum_{i=1, i \neq j}^{n} X_{i j} c_{i} c_{j}
$$

where: $X_{i j}=4 \Delta H_{\text {mix }} \mathrm{AB}$ where $\Delta H_{\text {mix }} \mathrm{AB}$ - mixing enthalpy of $\mathrm{AB}$ binary system, $c_{i}, c_{j}-$ molar ratio of $i$ and $j-$ elements.

In literature $[2,16,17]$ the high value of $\Delta S_{m i x}$ parameter is usually used for the stabilization of solid solutions by compensating the low value of $\Delta H_{m i x}$ in HEAs. For the equi-molar ratios for five-component alloys the values of $\Delta S_{m i x}$ should be approx. $13.4 \mathrm{~J} / \mathrm{mol} \cdot \mathrm{K}(1.6 \mathrm{R})$ [1], while for near equi-molar ratios $\Delta S_{m i x}$ value should be approx. $12.4 \mathrm{~J} / \mathrm{mol} \cdot \mathrm{K}(1.5 \mathrm{R})$. Such high values of the mixing entropy are expected to stabilize the $\mathrm{BCC}$ and/or FCC phase and prevent the formation of intermetallic phases. Three out of four analyzed alloys fulfil this condition (Table 2), however based on the XRD analysis, which revealed Laves-type phase and martensite-type phase, it is assumed that this condition is not sufficient enough to obtain the predicted FCC and/or BCC solid solutions. The mixing entropy should be compensate with mixing enthalpy. For multi-component solutions, the $\Delta H_{\text {mix }}$ value should be between $-15 \mathrm{~kJ} / \mathrm{mol}^{-1}$ and $5 \mathrm{~kJ} / \mathrm{mol}^{-1}$ [16]. The values calculated for three out of the four investigated alloys are more negative. According to the presented values it was concluded that the mixing entropy and mixing enthalpy do not compensate for each other. It should be noted that alloy 4 has $\Delta S_{m i x}$ value lower 

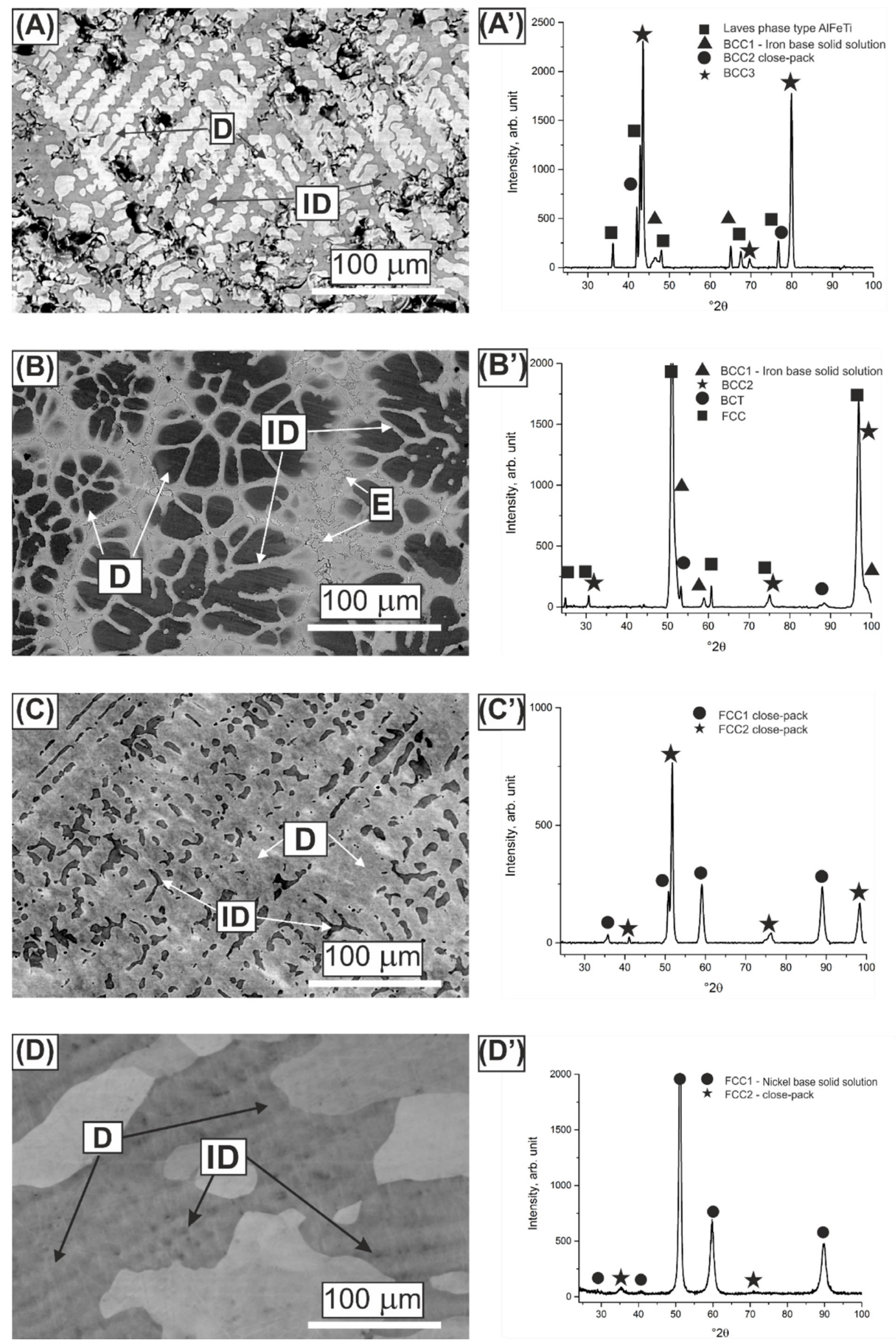

(D')

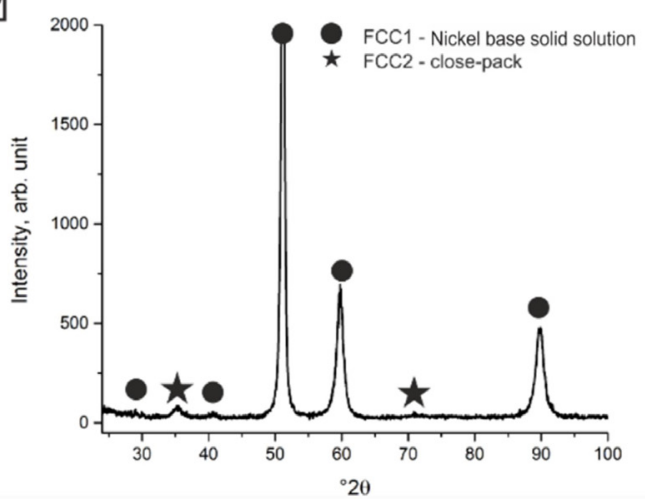

Fig. 1. Microstructure and XRD patterns of the investigated alloys: (A) and (A') - alloy 1, (B) and (B') alloy 2, (C) and (C') alloy 3, (D) and (D') - alloy 4. The Dendritic areas are signed as 'D', Interdendritic areas as 'ID' and Eutectic as 'E'. 


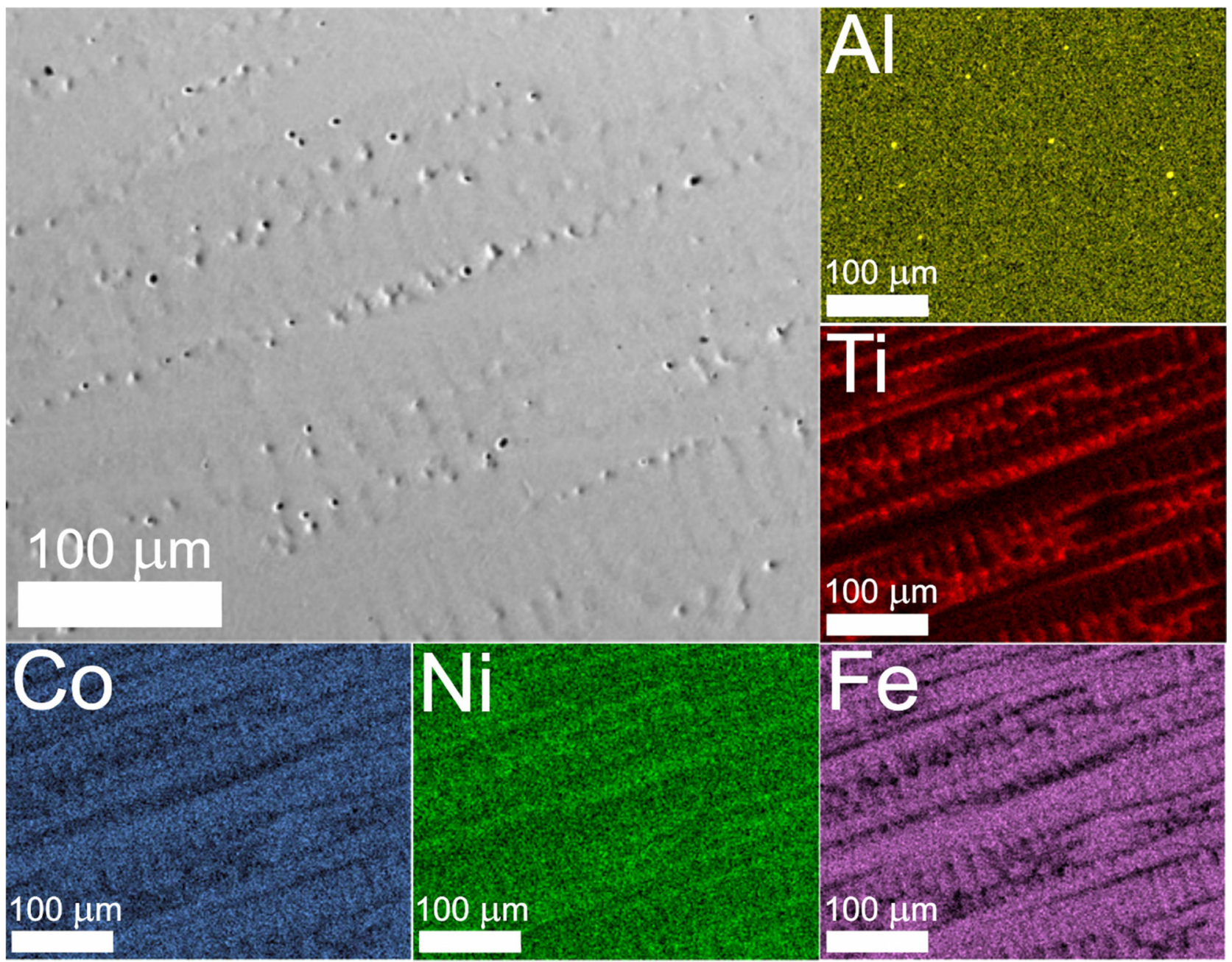

Fig. 2. EDS analysis of elements distribution in alloy 4

than $12.4 \mathrm{~J} / \mathrm{mol} \cdot \mathrm{K}$ but its $\Delta H_{m i x}$ value is less negative than for the remaining samples, additionally $\mathrm{Al}_{5} \mathrm{Ti}_{5} \mathrm{Co}_{35} \mathrm{Ni}_{35} \mathrm{Fe}_{20}$ is the only alloy that fulfils the $\Delta H_{\text {mix }}$ condition.

Other parameters that should be considered during designing phases are: $\delta$ (eq. 4 ) and $\Omega$ (eq. 5) parameters:

$$
\delta=100 \sqrt{\sum_{i=1}^{n} c_{i}\left(1-r_{i} / \bar{r}\right)^{2}}
$$

where: $\bar{r}=\sum_{i=1}^{n} c_{i} r_{i}, c_{i}-$ molar ratio, $r_{i}-$ atomic radius of each element.

$$
\Omega=\frac{T_{m} \Delta S_{m i x}}{\left|\Delta H_{m i x}\right|}
$$

where $T_{m}=\sum c_{i}\left(T_{m}\right)_{i},\left(T_{m}\right)_{i}-$ melting point of each element.

The $\delta$ parameter describes the differences of atomic sizes between the used alloying elements and the average value of the whole alloy. The solid solutions are expected to form for components which $\delta$ value is less than 6.6 pct. [16]. For higher values of $\delta$ the severe lattice distortion effect may be disrupted. Based on the calculated values (Table 3 ) it is visible that with strong negative $\Delta H_{m i x}$ values the value of the $\delta$ parameter increases.
The parameter which interconnects the relation between the mixing entropy and mixing enthalpy is the $\Omega$ parameter. For multi-component alloys, the $\Omega$ value should be greater than 1.1 [16]. Lower values will indicate that $\Delta S_{m i x}$ does not compensate for the $\Delta H_{m i x}$. Among all investigated alloys, only alloy 3 and 4 have $\Omega$ value greater than 1.1 .

TABLE 3

$\delta$ and $\Omega$ parameters of synthesized alloys

\begin{tabular}{|c|c|c|}
\hline \hline & $\boldsymbol{\delta}$ & $\boldsymbol{\Omega}$ \\
\hline Alloy 1 & 7.3 & 0.6 \\
\hline Alloy 2 & 7.4 & 0.8 \\
\hline Alloy 3 & 6.3 & 1.2 \\
\hline Alloy 4 & 4.8 & 2.0 \\
\hline
\end{tabular}

Based on the data analysis presented in Table 3, it is concluded that the solid solutions could be formed only when $\Delta H_{m i x}, \delta$ and $\Omega$ conditions (proper values of each parameter) are fulfilled. What is interesting is that even if the $\Delta S_{m i x}$ condition in not realized it is possible to form a solid solution (alloy 4). An example of this could be alloy 3 , where the $\Delta H_{m i x}$ value is more negative than the boundary value for the multi-component 
alloys. However, it should be emphasized that in general the $V E C$ parameter enables the prediction of the type of expected crystallographic structure, but it does not allow to design solid state solutions. It should be noticed than even if $\Delta H_{m i x}, \delta$ and $\Omega$ conditions are fulfilled it is possible that in the material in some systems could exist microsegregation [18].

\section{Conclusions}

The criterions that are most commonly used in designing and the synthesis of high-entropy alloys are valence electron concentration $(V E C)$ and high entropy of mixing $\left(\triangle S_{\text {mix }}\right)$. However, as presented in this paper, these two parameters are not sufficient enough to ensure the obtaining of solid solutions. In many circumstances the high value of mixing entropy is not able to compensate for the negative value of mixing enthalpy. The parameter which connects $\Delta S_{m i x}$ and $\Delta H_{m i x}$ is $\Omega$-values higher than 1.1 guarantee the most optimal conditions for FCC and/or BCC phase stabilization. Another parameter directly connected with mixing enthalpy is $\delta$, and its value should be lower than 6.6 pct. It seems that one of the most important factors which should be considered in the synthesis of HEAs is $\Delta H_{\text {mix }}$ with values falling between $-15 \mathrm{~kJ} / \mathrm{mol}^{-1}$ and $5 \mathrm{~kJ} / \mathrm{mol}^{-1}$. In some systems could exist microsegregation even if all parameters for solid solution are satisfy.

\section{Acknowledgements}

Research supported by Ministry of Science and Higher Education Republic of Poland (project No. 15.11.110.505)

\section{REFERENCES}

[1] J.W. Yeh, Y.L. Chen, S.J. Lin, S. K. Chen, Mater. Sci. Forum 560, 1-9 (2007).

[2] Y. Zhang, X. Yang, P.K. Liaw, Jom 64, 830-838 (2012).

[3] Y. Zhang, Y. Zhou, X. Hui, M. Wang, G. Chen, Sci. China Ser. G Physics, Mech. Astron. 51, $427-437$ (2008).

[4] M.J. Yao, K.G. Pradeep, C.C. Tasan, D. Raabe, Scr. Mater. 72-73, 5-8 (2014).

[5] Y.Y. Chen, T. Duval, U.D. Hung, J.W. Yeh, H.C. Shih, Corros. Sci. 47, 2257-2279 (2005).

[6] C. Hsu, J.W. Yeh, S. Chen, T. Shun, Metall. Mater. Trans. A 35A, 1465-1469 (2004).

[7] Y.F. Kao, T.J. Chen, S.K. Chen, J.W. Yeh, J. Alloys Compd. 488, 57-64 (2009).

[8] A.V. Kuznetsov, D.G. Shaysultanov, N.D. Stepanov, G.A. Salishchev, O.N. Senkov, Mater. Sci. Eng. A 533, 107-118 (2012).

[9] C.P. Lee, Y.Y. Chen, C.Y. Hsu, J.W. Yeh, H.C. Shih, J. Electrochem. Soc. 154, C424-C430 (2007).

[10] C. Li, J.C. Li, M. Zhao, Q. Jiang, J. Alloys Compd. 475, 752-757 (2009).

[11] X.F. Wang, Y. Zhang, Y. Qiao, G.L. Chen, Intermetallics 15, $357-$ 362 (2007).

[12] M.A. Hemphill, T. Yuan, G.Y. Wang, J.W. Yeh, C.W. Tsai, A. Chuang, P.K. Liaw, Acta Mater. 60, 5723-5734 (2012).

[13] D. Miracle, J. Miller, O. Senkov, C. Woodward, M. Uchic, J. Tiley, Entropy 16, 494-525 (2014).

[14] S. Guo, C. Ng, J. Lu, C. T. Liu, J. Appl. Phys. 109, 103505-1 (2011).

[15] Y.L. Chou, J.W. Yeh, H.C. Shih, Corros. Sci. 52, 2571-2581 (2010).

[16] X. Yang, Y. Zhang, Mater. Chem. Phys. 132, 233-238 (2012).

[17] S. Guo, Q. Hu, C. Ng, C.T. Liu, Intermetallics 41, 96-103 (2013).

[18] K. Górecki, P. Bała, G. Cios, T. Kozieł, M. Stępień, K. Wieczerzak, Metall. Mater. Trans. A 47A, 3257-3262 (2016). 